\title{
PREMIÈRES OBSERVATIONS SUR LA COEXISTENCE DE DREISSENA POLYMORPHA, COROPHIUM CURVISPINUM ET CORBICULA SPP. DANS LA RIVIERE MOSELLE.
}

\author{
V. BACHMANN (1), P. USSEGLIO-POLATERA (1, 2), E. CEGIELKA (1), \\ P. WAGNER (1), J.F. POINSAINT (1), J.C. MORETEAU (1).
}

(1) Equipe de Démoécologie, C.R.E.U.M., Université de Metz, 1 rue des Récollets, 57040 Metz Cedex 01, France.

(2) E.S.A. C.N.R.S. 5023, Ecologie des Eaux Douces et des Grands Fleuves, 43 Bd du 11 Novembre 1918, 69622 Villeurbanne Cedex, France.

\section{RÉSUMÉ}

La macrofaune benthique de la rivière Moselle a été échantillonnée par dragage sur plusieurs stations de son profil longitudinal, de juillet 1994 à septembre 1995. Ces dragages ont été réalisés suivant plusieurs transects, à des profondeurs de 1 à 4 mètres, sur chaque station. Ceux-ci ont permis de découvrir la présence de l'amphipode Corophium curvispinum et de deux formes (ou espèces?) différentes de bivalve du genre Corbicula. Ces recherches ont montré l'extension de l'aire de répartition de C. curvispinum à la Moselle française, jusqu'à Gondreville au moins (soit 137,5 km de cours nouvellement colonisé), et l'extension de l'aire de répartition du genre Corbicula jusqu'à Dieulouard (soit $337 \mathrm{~km}$ de cours depuis l'embouchure sur le Rhin). La distribution de ces espèces, réputées comme invasives et responsables de sérieux problèmes de "salissures" pour les utilisateurs d'eau brute des cours d'eau de plaine, semble être en relation avec la nature du substrat. Des phénomènes de compétition spatiale et trophique entre ces nouveaux arrivants et les populations macrobenthiques autochtones sont envisageables. Un suivi attentif de ces populations est nécessaire afin de prévenir d'éventuels problèmes économiques, voire écosystémiques.

\section{PRELIMINARY OBSERVATIONS ABOUT THE COEXISTENCE OF DREISSENA POLYMORPHA, COROPHIUM CURVISPINUM AND CORBICULA SPP. IN THE RIVER MOSELLE.}

\begin{abstract}
Benthic macroinvertebrates were sampled at a water depth of 1-4 metres by means of benthic dredges along several transects of the River Moselle from July 1994 to September 1995. These samples revealed the recent establishment of the amphipod Corophium curvispinum and of two forms (or species?) of the genus Corbicula (Bivalvia). Our sampling programme showed that the biogeographical distribution of $C$. curvispinum included the French part of the River Moselle at least up to Gondreville (i.e. $137.5 \mathrm{~km}$ newly colonized), and that Corbicula spp. colonized the River Moselle up to Dieulouard (i.e. $337 \mathrm{~km}$ from the confluence of the river with the Rhine). The distribution of these species, considered as mass invaders that may cause serious fouling problems for river water users, seems to be correlated with
\end{abstract}


characteristics of the substratum. Spatial and trophic competition between these new immigrants and previously established macrobenthic populations are possible. The structure and the dynamics of these populations must now be carefully observed, in order to prevent possible economic and ecosystemic damages.

\section{INTRODUCTION}

Dreissena polymorpha, communément appelée Moule d'eau douce ou Moule zébrée, est un bivalve lamellibranche, fixé par un byssus à l'état adulte, originaire de la région PontoCaspienne. Elle est connue pour l'extraordinaire extension de son aire de répartition géographique depuis 1800 . Cette espèce est, de nos jours, présente dans la majeure partie des lacs et rivières européens et considérée comme une constante de la macrofaune benthique (HEBERT et al., 1989 ; VAN DER VELDE et al., 1994), en particulier dans la rivière Moselle. Si sa propagation est largement expliquée par les activités humaines, et notamment par la construction d'importants réseaux de canaux ainsi que par l'intensification de la navigation, on notera cependant les importantes capacités d'adaptation de cette espèce, qui contribuent sans nul doute à son caractère invasif. Cette caractéristique explique que de nombreux biologistes de par le monde étudient non seulement ses caractéristiques biologiques (NALEPA et SCHLOESSER, 1993), mais aussi les divers moyens mécaniques, physiques, chimiques ou biologiques susceptibles de freiner ou d'endiguer son développement de masse. Il est à noter que si cette espèce a fait l'objet d'une telle attention, c'est bien évidemment suite aux nombreux problèmes qu'elle a occasionnés et occasionne encore aux industriels et distributeurs d'eau, en particulier au niveau de leurs installations (pertes de charge, colmatages des prises d'eau et conduites, augmentation de la corrosion...) (GREENSHIELDS et RIDLEY, 1957 ; NALEPA et SCHLOESSER, 1993).

L'amphipode Corophium curvispinum Sars, 1895 est, comme $D$. polymorpha, natif du bassin de la mer Caspienne et a étendu son aire géographique de répartition à la majorité des cours d'eau européens depuis le début du $20^{\text {eme }}$ siècle. Ce petit crustacé de 2,5 à $7 \mathrm{~mm}$ de long se reconnaît facilement par sa deuxième paire d'antennes hyper-développées. Il colonise le substrat en construisant un tube cylindrique dans lequel il vit et qui est formé de fils de soie auxquels des particules sableuses et limoneuses sont accolées. Les fils de soie sont produits par ses glandes sétifères. II tolère les conditions perturbantes créées par le batillage et utilise les eaux de ballast des bateaux comme moyen de dispersion (PYGOTT et DOUGLAS, 1989). L'espèce a été signalée dans la Meuse (WOUTERS, 1985), puis découverte pour la première fois dans le Rhin en 1987 où, deux ans plus tard, elle constitue la majeure partie du peuplement macrobenthique de la partie aval de ce fleuve (DEN HARTOG et al., 1992; PAFFEN et al., 1994). Depuis 1988, une population de plusieurs milliers d'individus par mètre carré était signalée à l'embouchure de la Moselle (SCHÖLL, 1990). Des individus avaient colonisé la partie germano-luxembourgeoise de cette rivière jusqu'à Remich (pK 229,5), considérée alors comme la limite amont de répartition géographique de cette espèce (DHUR et MASSARD, 1995).

Les bivalves lamellibranches du genre Corbicula sont originaires d'Asie, où ils composent une large part de la faune macrobenthique (MCMAHON, 1982). Corbicula fluminea a été introduite aux États-Unis au début du siècle et s'est répandue très rapidement sur le continent nord-américain, dans des proportions hors du commun. C'est en 1987 qu'elle fut découverte dans le Rhin, et KINZELBACH (1991) note que probablement deux espèces de Corbicula au moins ont émigré en Europe par l'intermédiaire des eaux de ballast des navires provenant du sud de l'Amérique du Nord et qu'il n'y aurait de ce fait aucun lien géographique direct avec les populations de Corbicula asiatiques. A l'heure actuelle, il apparait qu'une grande 
incertitude subsiste quant à la taxonomie des Corbicula présentes dans le Rhin et dans la Moselle. Suivant les auteurs, ce serait deux espèces : $C$. fluminea et $C$. fluviatilis (KINZELBACH, 1991 ; DEN HARTOG et al., 1992), ou deux écotypes différents : C. fluminea et $C$. fluminalis si on se réfère à la systématique revue par ZADHIN (1965), et MORTON $(1979,1986)$ (critères de distinction repris par BIJ DE VAATE et GREIJDANUS-KLAAS, 1990 ; HAESLOOP, 1992). Le genre Corbicula a été recensé par MOUTHON (1981) dans la rivière Dordogne (France) et dans le Tage (Portugal), dans l'estuaire de la Weser en Allemagne du Nord par HAESLOOP (1992), dans la partie hollandaise du Rhin par BIJ DE VAATE et GREIJDANUS-KLAAS (1990) et dans la rivière Miño en Espagne (ARAUJO et al., 1993). De récentes études montrent que C. fluminea a colonisé de nombreux cours d'eau du sud-ouest de la France (DUBOIS, 1995a, 1995b). MCMAHON (1983) considère C. fluminea aux États-Unis comme " the most important molluscan pest species ever introduced in the USA", sa prolifération ayant entrainé des dommages considérables aux installations hydrauliques des cours d'eau qu'elle colonisait. La biologie des espèces (ou écotypes) de Corbicula est essentiellement déduite des caractéristiques de populations américaines ou asiatiques. Elle est fort mal connue dans les conditions écologiques des rivières européennes. II apparaît cependant que $C$. fluminea et $C$. fluminalis auraient des caractéristiques biologiques très différentes (hermaphrodisme vs gonochorisme, longévité faible vs longévité forte, croissance rapide vs croissance lente, incubation des larves vs phase larvaire planctonique...) (McMAHON, 1983 ; MORTON, 1983, 1986, 1987). Ces différences n'ont pas été confirmées à ce jour dans les écosystèmes européens. Seules les caractéristiques biologiques de $C$. fluminea ont été récemment vérifiées dans le sud-ouest de la France par DUBOIS (1995b).

C'est en Juillet 1994 que nous avons recensé pour la première fois dans le cours français de la Moselle Corbicula spp. et C. curvispinum (BACHMANN et al., 1995). Connaissant les fortes densités de $D$. polymorpha dans cette rivière et les divers problèmes qu'elle a occasionnés au niveau des installations hydrauliques, nous avons essayé d'établir (1) les limites de répartition géographique et (2) une première cartographie des densités de populations de ces nouveaux arrivants dans la Moselle. Une éventuelle relation entre les effectifs des populations observées et la nature du substrat colonisé a également été recherchée. Notre étude, qui se poursuit, a notamment pour objectif de comprendre, d'expliquer, voire de prédire leur devenir dans cet écosystème.

\section{MATÉRIEL ET MÉTHODES}

La Moselle prend sa source au col de Bussang (Massif des Vosges) et se jette dans le Rhin à Coblence, après $535 \mathrm{~km}$ de cours dont $293 \mathrm{~km}$ en France. La confluence avec la Meurthe à Pompey (pK 345,08 ), de par la modification des caractéristiques physico-chimiques de l'eau qu'elle entraine, permet de distinguer la Moselle amont de la Moselle aval. Cette rivière, principalement dans sa partie aval, fait partie des grandes rivières régulées où l'activité fluviale a entraîné un aménagement conséquent du cours, et donc une artificialisation importante du milieu physique qui a contribué à une simplification de la mosaïque d'habitats benthiques. Les voies de navigation empruntent tantôt le cours même de la Moselle et tantôt des dérivations. On a donc un tressage de secteurs "sauvages" (= non canalisés) et de secteurs canalisés (= navigables) empruntant soit le cours de la Moselle, soit des canaux de dérivation.

L'acquisition des données faunistiques et mésologiques a été réalisée de juillet 1994 à septembre 1995, principalement en période estivale.

\section{Répartition géographique des espèces étudiées}

La détermination de l'aire de répartition géographique des trois espèces invasives a été réalisée sur huit stations, de Grevenmacher (Luxembourg - pK 211,0) à Gondreville (France - pK 367,0) (Figure 1). 


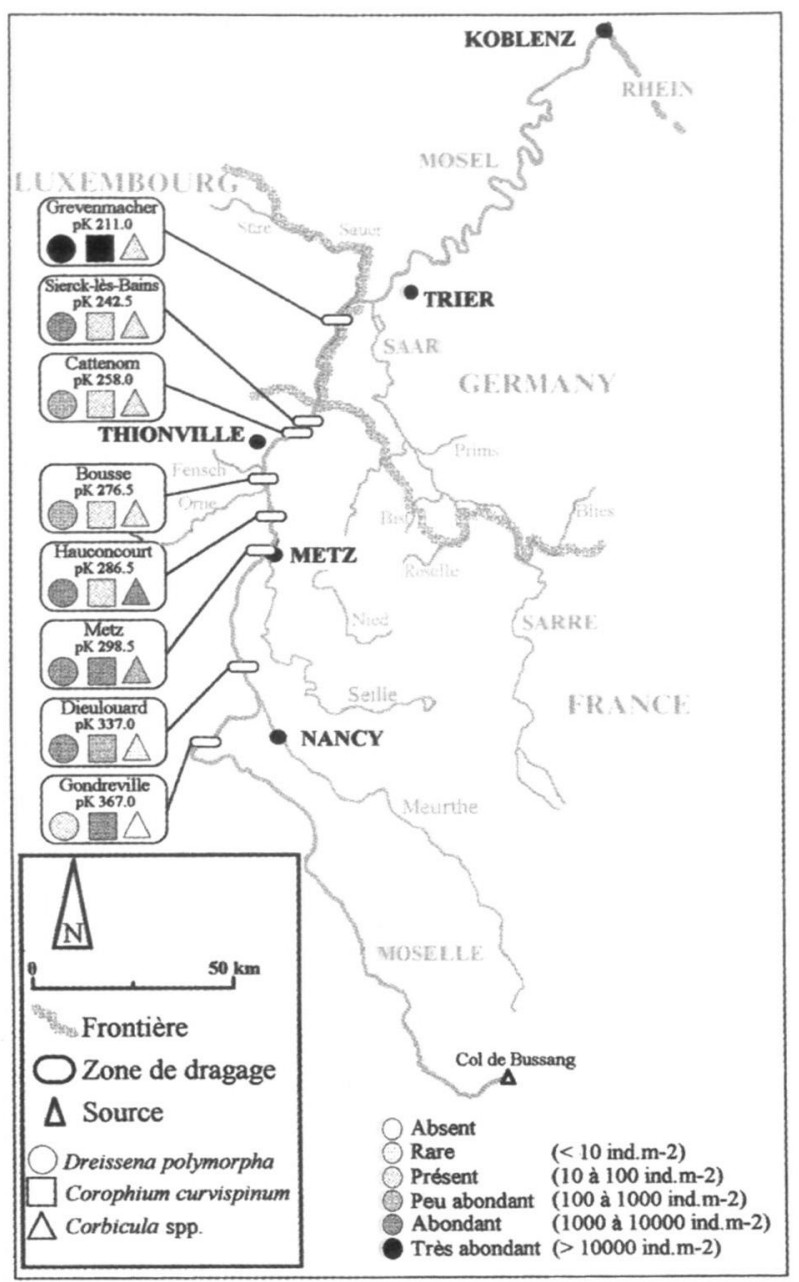

\section{Figure 1}

Carte de la répartition géographique et des densités moyennes au $\mathbf{m}^{2}$ de Dreissena polymorpha, Corophium curvispinum et Corbicula spp. dans la rivière Moselle. Les pK utilisés correspondent aux points kilométriques de navigation.

Figure 1

Map of the biogeographic distribution and of the mean densities per square metre of Dreissena polymorpha, Corophium curvispinum and Corbicula spp. in the River Moselle. pK represents navigation kilometric points.

Les composantes benthiques (minérale et biologique) sont échantillonnées par bateau, à des profondeurs variant de 1 à 4 mètres, au moyen de deux types de dragues, utilisés suivant la texture du substrat rencontré : une drague triangulaire, de $39 \mathrm{~cm}$ d'ouverture, et une drague cylindro-conique de type Rallier du Baty modifiée, de $30 \mathrm{~cm}$ de diamètre d'ouverture (décrites dans BERLY, 1989). Sur chaque station, le chenal et les deux rives sont prospectés. Compte tenu de la profondeur de travail connue des deux engins et de leur section, la surface échantillonnée lors de chaque dragage est estimée en adoptant la procédure préconisée par BERLY (1989). Les prèlèvements collectés sont récupérés dans des bidons de 2 litres et conservés dans de l'alcool à $70^{\circ}$. Le tri et le dénombrement des spécimens de $D$. polymorpha, Corbicula spp. et $C$. curvispinum sont réalisés au laboratoire.

\section{Étude des relations espèces/substrat}

L'étude des affinités de ces trois taxons pour différents types de substrat est réalisée sur les seules stations de Grevenmacher (pK 211,0 à 212,2) et Sierck-lès-Bains (pK 242,5), 
respectivement représentatives d'une zone fortement et moyennement artificialisée, et Hauconcourt (pK 286,1 à 287,5), représentative d'une zone " sauvage ".

\section{Acquisition des données faunistiques et granulométriques}

La méthode de prélèvement est identique à celle décrite précédemment. Suite à l'évaluation des densités spécifiques, la structure granulométrique de chaque prélèvement, alors exempt de la composante biologique, est caractérisée par la détermination des poids secs (après passage à l'étuve à $105{ }^{\circ} \mathrm{C}$ pendant 24 heures) de dix fractions granulométriques successives (] $0-0,5 \mathrm{~mm}]=$ limons $;] 0,5-1 \mathrm{~mm}]=$ sables fins $;] 1-2 \mathrm{~mm}]=$ sables grossiers ; ]2-4 mm] = graviers fins ; ]4-8 mm] = graviers moyens ; ]8-16 mm] = graviers grossiers ; ]16-32 $\mathrm{mm}]=$ petits cailloux ; $] 32-64 \mathrm{~mm}]=$ cailloux ; $] 64-128 \mathrm{~mm}]=$ pierres ; $] 128 \mathrm{~mm}-\infty[=$ blocs $)$. Le substrat " terre" (essentiellement constitué de particules argileuses et de matières organiques finement divisées) échantillonné sur la station de Sierck-lès-Bains est considéré comme un type de substrat particulier. La structure granulométrique d'un relevé est définie par l'abondance relative estimée (en terme de masse) de chacune des fractions granulométriques identifiée au sein du substrat.

\section{Traitement des données}

Les masses relatives des différentes classes granulométriques obtenues pour chaque relevé sont exprimées de manière semi-quantitative suivant un gradient croissant (Tableau I).

\section{Tableau I}

Variables (11 classes granulométriques) et modalités utilisées dans la description de la structure mésologique des relevés.

Table 1

Variables (eleven granulometric classes) and modalities used for the description of the mesological structure of samples.

\begin{tabular}{|c|c|c|c|c|c|c|}
\hline \multirow{2}{*}{ Variables } & \multicolumn{3}{|c|}{ Modalités } & Maximum \\
\cline { 2 - 6 } & 0 & 1 & 2 & 3 & 4 & rencontré \\
\hline sables fins & absence & ] $0 \% ; 5 \%[$ & {$[5 \% ; 10 \%[$} & $\geq 10 \%$ & & $26 \%$ \\
sables grossiers & absence & $10 \% ; 5 \%[$ & {$[5 \% ; 10 \%[$} & $\geq 10 \%$ & & $35 \%$ \\
graviers fins & absence & $10 \% ; 5 \%[$ & {$[5 \% ; 10 \%[$} & $\geq 10 \%$ & & $18 \%$ \\
graviers moyens & absence & $10 \% ; 5 \%[$ & {$[5 \% ; 10 \%[$} & $\geq 10 \%$ & & $14 \%$ \\
graviers grossiers & absence & ] $0 \% ; 5 \%[$ & {$[5 \% ; 10 \%[$} & {$[10 \% ; 16 \%[$} & $\geq 16 \%$ & $23 \%$ \\
petits cailloux & absence & $10 \% ; 10 \%[$ & {$[10 \% ; 20 \%[$} & $\geq 20 \%$ & & $37 \%$ \\
cailloux & absence & ] $0 \% ; 10 \%[$ & {$[10 \% ; 20 \%[$} & $\geq 20 \%$ & & $85 \%$ \\
galets & absence & ] $0 \% ; 5 \%[$ & {$[5 \% ; 10 \%[$} & {$[10 \% ; 20 \%[$} & $\geq 20 \%$ & $78 \%$ \\
blocs & absence & ] $0 \% ; 50 \%[$ & $\geq 50 \%$ & & & $98 \%$ \\
terre & absence & présence & & & \\
\hline
\end{tabular}


Une Analyse des Correspondances Multiples est appliquée au tableau (27 relevés $\times 44$ modalités de 11 variables granulométriques). Elle permet de comparer la structure granulométrique des relevés des différentes stations. La projection des abondances faunistiques des trois taxons considérés sur les positions des relevés dans le plan $F_{1}-F_{2}$ de l'A.C.M. illustre les affinités de chaque taxon pour une certaine structure granulométrique de l'habitat. La comparaison de ces affinités peut permettre de préciser sur quels types de substrat une compétition entre certaines de ces espèces est à envisager. Les calculs et les représentations graphiques ont été réalisés à l'aide du logiciel A.D.E. 4.0. (CHESSEL et DOLEDEC, 1995).

\section{RÉSULTATS}

\section{Distribution longitudinale des espèces}

Une première carte de répartition des trois espèces invasives étudiées dans la Moselle a été dressée en 1995 (Figure 1). Au regard des densités moyennes calculées et figurées en classes d'abondance sur chaque station, il apparait que si $D$. polymorpha et $C$. curvispinum sont présentes jusqu'au niveau du point le plus amont étudié, à savoir Gondreville, Corbicula spp. n'a pas été récoltée dans cette station. A l'heure actuelle, on peut penser que la limite géographique de répartition de ce genre se situerait aux alentours de Dieulouard (pK 337,0 ). D'autre part, l'abondance de C. curvispinum à Gondreville laisse supposer que cette espèce est certainement présente bien à l'amont de cette station. II ne lui aura donc fallu que deux ans au plus pour étendre son aire de répartition de Remich (DHUR et MASSARD, 1995) à Gondreville, ce qui correspond à $137,5 \mathrm{~km}$ de cours.

Si on considère séparément la distribution de chaque espèce sur l'ensemble des stations étudiées, les densités moyennes observées ne montrent aucun gradient longitudinal amontaval. Une différence de structure de la mosaïque de substrats rencontrée sur les sites d'étude prospectés pourrait éventuellement expliquer une absence de gradient.

\section{Les relations espèces/substrat}

L'Analyse des Correspondances Multiples appliquée au tableau mésologique met en évidence les différences de structure granulométrique des trois stations échantillonnées (Figure 2).

Les deux premiers axes factoriels retenus expliquent $40 \%$ de la variance du tableau initial (Figure 2a). L'axe 1 montre principalement l'opposition entre les caractéristiques granulométriques des stations de Hauconcourt et de Grevenmacher (Figure 2b). L'extrême homogénéité des positions des 7 relevés de Grevenmacher dans le plan $F_{1}-F_{2}$ est expliquée sur la Figure 2c par la forte dominance des blocs (modalité 3 ) dans tous les relevés toujours associés à une faible proportion de limons. A hauteur de cette station canalisée, les berges sont en effet constituées d'enrochements et le lit d'un pavage particulièrement homogène de gros blocs. La plupart des classes granulométriques (limons à galets) sont représentées dans les relevés de la station de Hauconcourt, illustrant ainsi la plus grande diversité du substrat de ce secteur sauvage et donc une richesse potentiellement plus grande en habitats benthiques.

La position intermédiaire sur l'axe 1 de la station de Sierck-lès-Bains (entre Grevenmacher et Hauconcourt - Figure 2b) se justifie par la présence d'un substrat particulier " terre " en rive droite, alors que les prélèvements réalisés dans le chenal et en rive gauche présentent une composition granulométrique plus hétérogène, proche de celle des relevés d'Hauconcourt. L'axe 2 (Figure 2) structure essentiellement les prélèvements de la station d'Hauconcourt, en opposant les relevés présentant les plus fortes teneurs en fines granulométries (qui correspondent aux modalités 3, 4 et/ou 5 des classes granulométriques " limon " à " petits cailloux ", au pôle positif de l'axe 2) aux relevés de granulométrie plus grossière (associés aux modalités 4 ou 5 des classes granulométriques "cailloux " et "galets ", au pôle négatif de l'axe 2). 

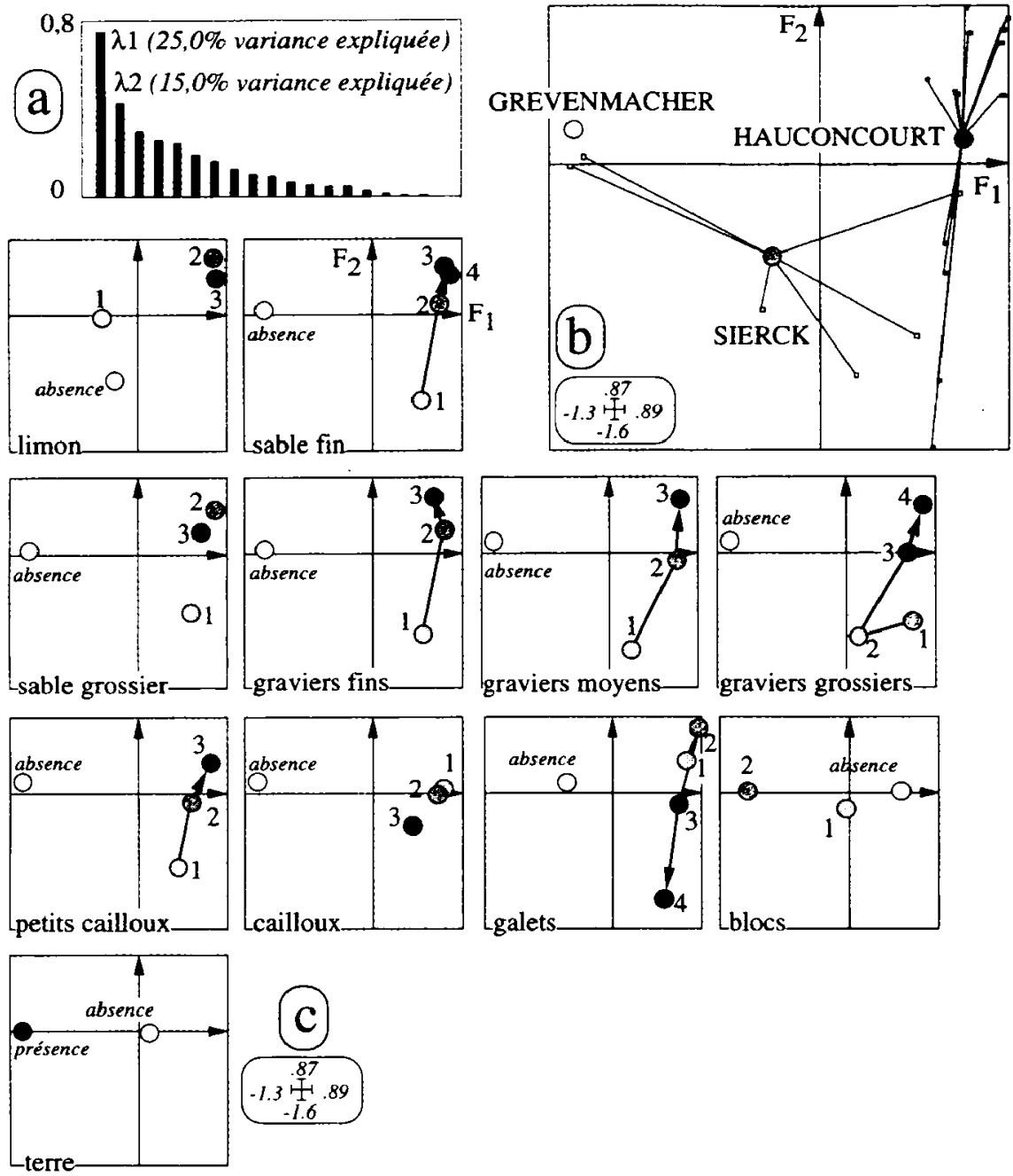

Figure 2

Résultats de l'Analyse des Correspondances Multiples réalisée sur le tableau des caractéristiques granulométriques des 27 relevés.

a. Histogramme des valeurs propres.

b. Distribution des stations sur le plan factoriel $F_{1}-F_{2}$. Chaque station (cercle) est positionnée au centre de gravité de ses échantillons (petits carrés). Chaque échantillon est relié à la station à laquelle il appartient par une ligne.

c. Distribution des modalités des 11 variables granulométriques dans le plan factoriel $F_{1}-F_{2}$. Chaque modalité est située au centre de gravité des échantillons qui l'utilisent. Le tableau I fournit la signification des modalités des 11 classes granulométriques.

Figure 2

Results of the Multiple Correspondence Analysis performed on the mesological data set.

a. Histogram of eigenvalues.

b. Distribution of stations (circles) on the $F_{1}-F_{2}$ factorial plane. They are located at the weighted average of their samples (small squares). Each sample is connected to its station circle by a line.

c. Distribution of modalities of the eleven granulometric variables on the $F_{1}-F_{2}$ factorial plane. Modalities are at the weighted average of the samples that present these modalities. See Table I for further details on modalities. 
Les densités de Corbicula spp., C. curvispinum et $D$. polymorpha observées dans les différents relevés sont ensuite projetées sur le plan $F_{1}-F_{2}$ des relevés, positionnés d'après leur structure granulométrique (Figure 3). Les 7 relevés de la station Grevenmacher ayant la même position sur le plan $F_{1}-F_{2}$, les densités faunistiques qui leur sont associées sont présentées de façon décalée sur la Figure 3, pour plus de clarté.

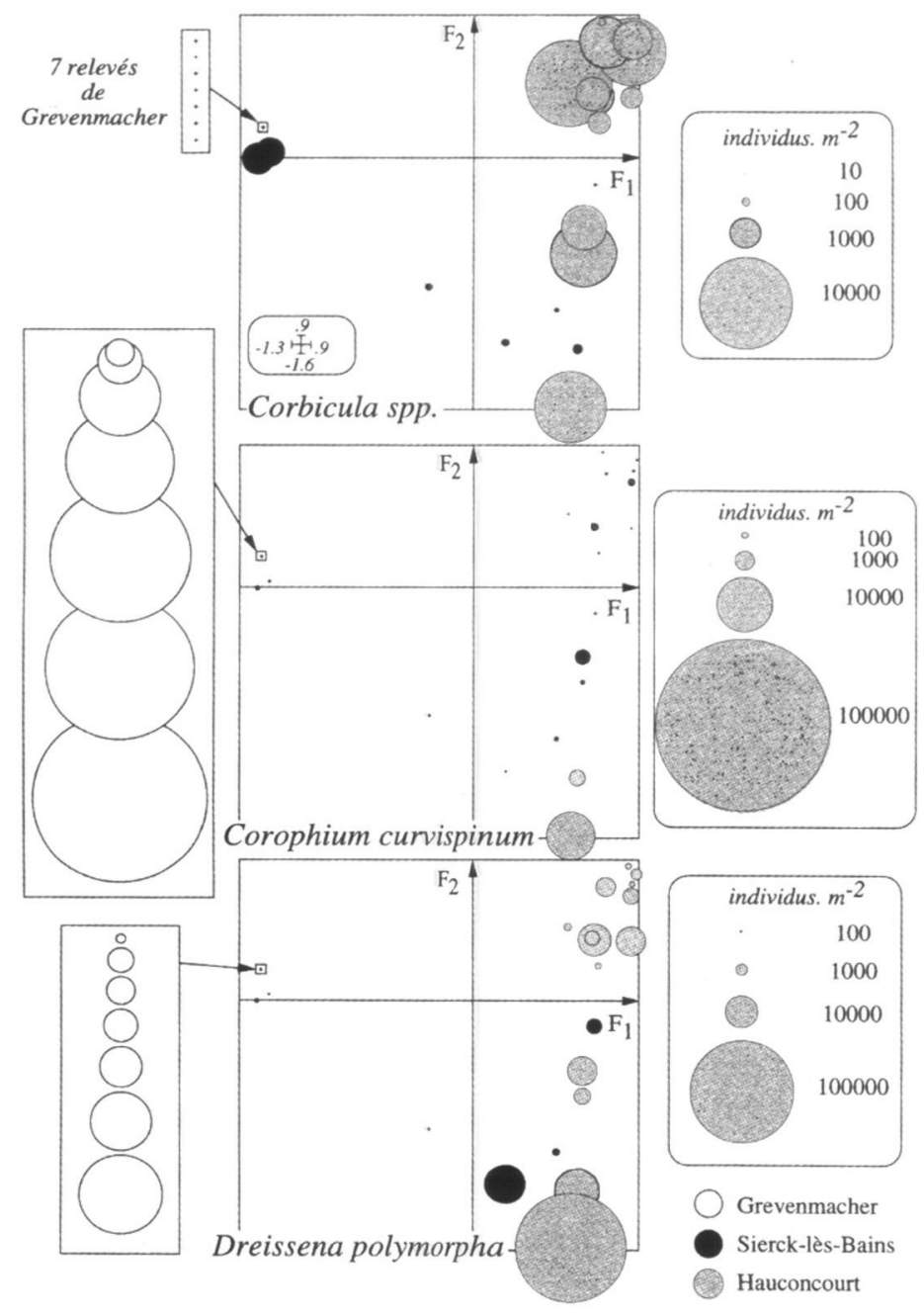

\section{Figure 3}

Projection des abondances spécifiques (cercles) des trois espèces invasives Corbicula spp., Corophium curvispinum et Dreissena polymorpha sur le plan factoriel $F_{1}-F_{2}$ des échantillons issu de l'Analyse des Correspondances Multiples appliquée au tableau mésologique. La taille des cercles est proportionnelle à l'abondance des espèces dans les échantillons. Les relevés de Grevenmacher ont une position identique ( $=$ croix encadrée) sur le plan $F_{1}-F_{2}$ de l'analyse. L'abondance relative de chaque taxon dans ces 7 relevés fait donc l'objet d'une représentation particulière. Une croix indique l'absence de l'espèce considérée dans le relevé correspondant.

Figure 3

Projection of the species abundances (circles) of the invaders Corbicula spp., Corophium curvispinum and Dreissena polymorpha on the $F_{1}-F_{2}$ factorial plane of the Multiple Correspondence Analysis performed on the mesological data set. The size of the circles is proportional to the species abundance in each sample. The samples of Grevenmacher exhibit the same location (the framed cross) on the $F_{1}-F_{2}$ factorial plane. So the relative abundances of each taxon in these samples are detailed in a particular figure. A cross indicates the absence of a species in a sample. 
II apparaît que les Corbiculidae sont quasi-absents des stations de Grevenmacher et de Sierck-lès-Bains, dans les prélèvements de granulométrie grossière (cailloux, galets, blocs) dominante. Leur affinité pour les substrats fins, en accord avec les observations de DEN HARTOG et al. (1992), est très marquée, notamment sur la station de Hauconcourt (densité maximale d'environ 9000 individus. $\mathrm{m}^{-2}$ ). A l'inverse, les plus fortes densités de $C$. curvispinum observées correspondent aux substrats de granulométrie grossière, principalement les blocs. Ainsi, cette espèce s'impose déjà à Grevenmacher comme le taxon dominant de la macrofaune, avec une densité atteignant près de 100000 individus. $\mathrm{m}^{-2}$. D. polymorpha est présente en densités importantes sur les trois stations étudiées et semble moins inféodée que c. curvispinum aux zones caractérisées par une dominance des fractions granulométriques grossières, ceci probablement du fait d'une installation plus ancienne de ses populations et/ou à des facultés d'adaptation plus importantes. Il est ainsi possible de trouver des grappes de $D$. polymorpha dans des zones de substrat essentiellement de fine granulométrie. En effet, la simple présence de quelques petits cailloux suffit à l'ancrage initial d'un petit nombre d'individus de $D$. polymorpha, qui serviront de “ substrat " à d'autres individus, comme l'a déjà signalé LEPAGE (1993, in NALEPA et SCHLOESSER, 1993).

\section{DISCUSSION}

Les eaux de la Moselle aval présentent une minéralisation élevée, par suite des apports salés de deux de ses affluents : la Meurthe et la Seille, respectivement affectées par la nature géologique du lit et par les rejets des soudières de Lorraine. Ces caractéristiques font de la Moselle une zone favorable à l'installation d'espèces d'origine estuarienne comme Corbicula spp. et $C$. curvispinum. Une extension rapide de leurs aires de répartition est d'ailleurs observée dans ce cours d'eau. Des densités particulièrement importantes sont alors rencontrées dans les habitats les plus favorables pour ces espèces.

La coexistence récente de $D$. polymorpha, C. curvispinum et Corbicula spp. n'est pas sans poser un certain nombre de questions quant au devenir des populations de ces trois taxons, compte tenu de leurs fortes potentialités colonisatrices respectives et de la nature des habitats et des sources nutritives disponibles. La possibilité d'une compétition spatiale entre $D$. polymorpha et $C$. curvispinum dans la Moselle apparaît clairement compte tenu de la présence de fractions granulométriques grossières, favorables à leur développement sur la majeure partie du cours canalisé. L'hypothèse d'une compétition tournant à l'avantage du crustacé peut éventuellement être envisagée si on se réfère aux observations faites sur le Rhin inférieur par VAN DER VELDE et al. (1994) illustrant l'important déclin des populations de $D$. polymorpha parallèlement à l'apparition massive de $C$. curvispinum sur une période de seulement six années. Les auteurs justifient cette évolution par le taux de reproduction très élevé de $C$. curvispinum, qui développe trois générations par an contre une seulement pour $D$. polymorpha. Les substrats durs sont donc rapidement colonisés et recouverts en abondance de tubes sablo-limoneux édifiés par $C$. curvispinum. Ils deviennent alors impropres à la fixation des larves de $D$. polymorpha, qui exigent également un substrat dur pour pouvoir se fixer.

C. curvispinum a une alimentation composée de diatomées, de petites chlorophycées ainsi que de détritus organiques (VAN DEN BRINK et al., 1993). Du fait de ses fortes densités, cette espèce a un certain impact sur le stock planctonique et entre donc logiquement en compétition trophique avec les filtreurs vrais que sont $D$. polymorpha (DEN HARTOG et al., 1992) et Corbicula spp., caractérisés par une activité de filtration importante. De plus, par la production de pseudo-fèces, $D$. polymorpha peut enrichir le milieu en débris organiques, et ainsi favoriser l'installation de C. curvispinum.

Outre l'aspect d'une compétition trophique entre ces trois espèces, leur présence simultanée et massive pourrait exercer une pression non négligeable sur le stock phytoplanctonique, et donc sur la production primaire de la rivière, déjà très perturbée en période estivale (GARNIER et BILLEN, 1995). 
Le Rhin est la voie d'immigration la plus vraisemblable permettant d'expliquer l'arrivée de C. curvispinum et Corbicula spp. dans la Moselle (SCHÖLL, 1990 ; DHUR et MASSARD, 1995). Cette invasion s'inscrit donc dans le cadre des introductions accidentelles d'espèces, imputables en majeure partie au transport fluvial qu'elles utilisent comme moyen de dispersion. Outre le fait que ces nouvelles espèces peuvent avoir des conséquences sur la structure de la communauté benthique autochtone et sur l'équilibre écologique de la rivière, c'est pour les utilisateurs d'eau brute que ces nouvelles espèces représentent un danger du même type que celui déjà occasionné par $D$. polymorpha (NALEPA et SCHLOESSER, 1993). Les jeunes individus de Corbicula, de faible taille, peuvent par exemple pénétrer dans les circuits de prise d'eau brute dans lesquels ils vont croître et se développer, jusqu'à éventuellement bloquer les systèmes de circulation d'eau de par leur masse propre ou de part la masse des matériaux et/ou déchets biologiques issus de leur développement massif. Par conséquent, face aux risques de dommages et problèmes sérieux que pourraient entraîner ces organismes invasifs, au niveau des installations de prise d'eau notamment, on ne peut que recommander aux utilisateurs d'eau brute une surveillance accrue de leurs populations au niveau de leurs sites. La mise en place de filtres adaptés à la collecte des larves (et/ou adultes) d'organismes invasifs, au niveau des prises d'eau, pourrait constituer un moyen pratique et efficace, mais sans doute de maintenance coûteuse, pour empêcher ou freiner la pénétration de ces espèces dans les circuits.

\section{CONCLUSIONS}

L'immigration de C. curvispinum et Corbicula spp. dans la Moselle est récente et la répartition biogéographique de ces nouvelles espèces invasives n'est probablement pas encore stabilisée dans le Nord-Est de la France. Il apparaît que $D$. polymorpha et $C$. curvispinum présentent une affinité commune pour un même type de substrat et un phénomène de compétition spatiale est envisageable. Cette compétition n'est pas encore démontrée dans la Moselle et seul un suivi à plus ou moins long terme des populations naturelles en relation avec les caractéristiques mésologiques de l'habitat et une étude expérimentale en conditions contrôlées permettront de la démontrer.

Compte tenu de l'intense trafic sur le dense réseau européen de voies de communications fluviales, il paraît impossible d'empêcher l'introduction accidentelle de nouvelles espèces à caractère potentiellement invasif si l'environnement s'avère favorable à leur développement. Pour le moins, peut-on souhaiter et/ou conseiller une plus grande attention quant au devenir des populations introduites.

\section{BIBLIOGRAPHIE}

ARAUJO R., MORENO D., RAMOS M.A., 1993. The Asiatic clam Corbicula fluminea (Müller, 1774) (Bivalvia : Corbiculidae) in Europe. Am. Malacol. Bull., 10, 39-49.

BACHMANN V., CEGIELKA E., WAGNER P., USSEGLIO-POLATERA P., MORETEAU J.C., 1995. Installation de l'amphipode Corophium Curvispinum et de la palourde asiatique Corbicula sp. dans le cours français de la Moselle. Hydroécol. Appl., 7 (1-2), (sous presse).

BERLY A., 1989. Distribution spatio-temporelle des peuplements macrobenthiques prélevés par dragage dans une station du Haut-Rhône. Thèse de doctorat, Université Lyon I, 309 p.

BIJ DE VAATE A., GREIJDANUS-KLAAS M., 1990. The Asiatic clam, Corbicula fluminea Müller, 1774 (Pelecypoda, Corbiculidae), a new immigrant in the Netherlands. Bull. Zool. Mus. Amst., 12 (12), 173-178.

CHESSEL D., DOLEDEC S., 1995. A.D.E. Software. Multivariate Analyses and Graphical Display for Environmental Data (version 3.7). Vol. 1 : User's Manual, Vol. 2 : Examples, Vol. 3 : Graphics Documentation, Université Lyon I, 523 p. 
DEN HARTOG C., VAN DEN BRINK F.W.B., VAN DER VELDE G., 1992. Why was the invasion of the River Rhine by Corophium curvispinum and Corbicula species so successful ? J. Nat. Hist., 26, 1121-1129.

DHUR G., MASSARD J.A., 1995. Etude historique et faunistique des invertébrés immigrés ou introduits dans la Moselle luxembourgeoise et ses affluents. Bull. Soc. Nat. Luxemb., 96 , 127-156.

DUBOIS C., 1995a. Corbicula fluminea : un mollusque opportuniste. Rev. Agence de l'Eau Adour-Garonne, 63, 13-16.

DUBOIS C., 1995b. Biologie et démo-écologie d'une espèce invasive Corbicula fluminea (Mollusca : Bivalvia) originaire d'Asie : étude en milieu naturel (canal à la Garonne, France) et en canal expérimental. Thèse de doctorat, Université Paul Sabatier, 169 p.

GARNIER J., BILLEN G., 1995. Bilan d'oxygène et processus biologiques dans les rivières régulées Moselle et Sarre. Rapport d'étude à la demande des Commissions Internationales pour la Protection de la Moselle et de la Sarre contre la pollution, Université Paris VI, CNRS URA 1367, 45 p.

GREENSHIELDS F., RIDLEY J.E., 1957. Some researches on the control of mussels in water pipes. J. Inst. Wat. Engrs, 11, 300-306.

HAESLOOP U., 1992. Establishment of the Asiatic clam Corbicula cf. fluminalis in the Tidal Weser River (N. Germany). Arch. Hydrobiol., 126 (2), 175-180.

HEBERT P.N.D., MUNCASTER B.W., MACKIE G.L., 1989. Ecological and genetic studies on Dreissena polymorpha (Pallas) : a new mollusc in the Great Lakes. Can. J. Fish. aquat. Sci., 46, 1587-1591.

KINZELBACH R., 1991. Die Körbchenmuscheln Corbicula fluminalis, Corbicula fluminea und Corbicula fluviatilis in Europa (Bivalvia : Corbiculidae). Mainz Natwiss. Archiv, 29, 215-228.

McMAHON R.F., 1982. The occurrence and spread of the introduced Asiatic freshwater clam, Corbicula fluminea (Müller) in North America ; 1924-1981. The Nautilus, 96, 134-141.

MCMAHON R.F., 1983. Ecology of the invasive pest bivalve Corbicula. In RUSSEL-HUNTER W.D., The Mollusca : Ecology, 6, 505-561, Academic Press, New-York.

MORTON B., 1979. Corbicula in Asia. In BRITTON J.C. (ed.), Proc. first Int. Corbicula Symp., 15-38, Texas Christian Univ., Fort Worth, Texas USA.

MORTON B., 1983. The sexuality of Corbicula fluminea (Müller) in lentic and lotic waters in Hong-Kong. J. Molluscan Stud., 49 (1), 81-83.

MORTON B., 1986. Corbicula in Asia : an updated synthesis. In BRITTON J.C. (ed.), Proc. second Int. Corbicula Symp., Am. Malacol. Bull., Spec. Publ. n², 113-124.

MORTON B., 1987. Polymorphism in Corbicula fluminea (Bivalvia : Corbiculidae) from Hong-Kong. Malacol. Rev., 20, 105-127.

MOUTHON J., 1981. Sur la présence en France et au Portugal de Corbicula (Bivalvia, Corbiculidae) originaire d'Asie. Basteria, 45, 109-116.

NALEPA T.F., SCHLOESSER D.W., 1993. Zebra Mussels : biology, impacts and control. Boca Raton, USA, Lewis Publishers, $810 \mathrm{p}$.

PAFFEN B.G.P., VAN DEN BRINK F.W.B., VAN DER VELDE G., BIJ DE VAATE A., 1994. The population explosion of the amphipod Corophium curvispinum in the Dutch Lower Rhine. Water Sci. Technol., 29 (3), 53-55. 
PYGOTT J.R., DOUGLAS S., 1989. Current distribution of Corophium curvispinum Sars var. devium Wundsch (Crustacea, Amphipoda) in Britain with notes on its ecology in the Shropshire Union Canal. Naturalist, 114, 15-17.

SCHÖLL F., 1990. Zur Bestandssituation von Corophium curvispinum Sars im Rheingebiet. Lauterbornia, 5, 67-70.

VAN DEN BRINK F.W.B., VAN DEN VELDE G., BIJ DE VAATE A., 1993. Ecological aspects, explosive range extension and impact of a mass invader, Corophium curvispinum Sars, 1895 (Crustacea : Amphipoda), in the Lower Rhine (The Netherlands). Oecologia, 93, 224-232.

VAN DER VELDE G., PAFFEN B.G.P., VAN DEN BRINK F.W.B., 1994. Decline of zebra mussel populations in the Rhine : competition between two mass invaders (Dreissena polymorpha and Corophium curvispinum). Naturwiss., 81, 32-34.

WOUTERS K.A., 1985. Corophium curvispinum SARS, 1895 (Amphipoda) in the River Meuse, Belgium. Crustaceana, 48 (2), 218-220.

ZADHIN V.I., 1965. Mollusks of fresh and brackish waters of the USSR [translated from Russian]. Academy of Sciences of the USSR, $368 \mathrm{p}$. 